\title{
Superiority of constructive interference in steady-state MRI sequencing over T1-weighted MRI sequencing for evaluating cavernous sinus invasion by pituitary macroadenomas
}

\author{
Min Lang, MS, ${ }^{1,3}$ Danilo Silva, MD, ${ }^{3-5}$ Lu Dai, MS, ${ }^{2}$ Varun R. Kshettry, MD, ${ }^{2-5}$ \\ Troy D. Woodard, MD, ${ }^{3-5}$ Raj Sindwani, MD, ${ }^{3-5}$ and Pablo F. Recinos, MD ${ }^{2-5}$ \\ ${ }^{1}$ School of Medicine, Case Western Reserve University; ${ }^{2}$ Cleveland Clinic Lerner College of Medicine, Case Western Reserve \\ University, Cleveland Clinic; ${ }^{3}$ Department of Neurosurgery, Neurological Institute, ${ }^{4}$ Minimally Invasive Cranial Base and Pituitary \\ Surgery Program, Rose Ella Burkhardt Brain Tumor \& Neuro-Oncology Center, ${ }^{5}$ Section of Rhinology, Sinus, and Skull Base \\ Surgery, Head and Neck Institute, Cleveland Clinic, Cleveland, Ohio
}

OBJECTIVE Preoperatively determining the extent of parasellar invasion of pituitary macroadenomas is useful for surgical planning and patient counseling. Here, the authors compared constructive interference in steady state (CISS), a T2weighted gradient-echo MRI sequence, to volume-interpolated breath-hold examination (VIBE), a T1-weighted gradientecho MRI sequence, for evaluation of cavernous sinus invasion (CSI) by pituitary macroadenomas.

METHODS VIBE and CISS images of 98 patients with pituitary macroadenoma were retrospectively analyzed and graded using the modified Knosp classification. The Knosp grades were correlated to surgical findings of CSI, which were determined intraoperatively using $0^{\circ}$ and $30^{\circ}$ endoscopes. The predictive accuracies for CSI according to the Knosp grades derived from the CISS and VIBE images were compared using receiver operating characteristic (ROC) curves. Postoperative MRI was used to evaluate the gross-total resection (GTR) rates.

RESULTS The CSI rate by pituitary macroadenomas was $27.6 \%$ (27 of 98 cases). Of 196 assessments (left and right sides of 98 macroadenomas), 45 (23.0\%) had different Knosp grades when scored using VIBE versus CISS images. For the VIBE images, $0 \%$ of Knosp grade $0,4.5 \%$ of grade $1,23.8 \%$ of grade $2,42.1 \%$ of grade $3 \mathrm{~A}, 100 \%$ of grade $3 \mathrm{~B}$, and $83.3 \%$ of grade 4 macroadenomas were found to have CSI intraoperatively. For the CISS images, $0 \%$ of Knosp grade 0 , $2.1 \%$ of grade $1,31.3 \%$ of grade $2,56.3 \%$ of grade $3 \mathrm{~A}, 100 \%$ of grade $3 \mathrm{~B}$, and $100 \%$ of grade 4 macroadenomas were found to have CSI intraoperatively. Two pituitary macroadenomas were classified as grade 4 on VIBE sequences but grades $3 \mathrm{~A}$ and 2 on CISS sequences; CSI was not observed intraoperatively in both cases. The GTR rate was $64.3 \%$ and $60.0 \%$ for high-grade (3A, 3B, and 4 ) macroadenomas classified using VIBE and CISS sequences, respectively. The areas under the ROC curves were 0.94 and 0.97 for VIBE- and CISS-derived Knosp grades ( $p=0.007$ ), respectively.

CONCLUSIONS Knosp grades determined using CISS sequence images are better correlated with intraoperative CSI than those determined using VIBE sequence images. CISS sequences may be valuable for the preoperative assessment of pituitary macroadenomas.

https://thejns.org/doi/abs/10.3171/2017.9.JNS171699

KEY WORDS volume interpolated breath-hold examination; VIBE; Knosp grade; endoscopic pituitary surgery; transsphenoidal surgery; CISS; constructive interference in steady state

AVERNOUs sinus invasion (CSI) occurs in 6\%-10\% of cases of pituitary adenomas and is one of the most common reasons for incomplete resection. ${ }^{1,12}$ The rate of gross-total resection (GTR) decreases from $78 \%$ to $92 \%$ to $20 \%$ to $52 \%$ when parasellar invasion is present. ${ }^{9,12,13,15,18,20,30,31}$ CSI can be predicted on radiological images, which aid the surgeon in preoperative planning. Total encasement of the internal carotid artery (ICA) is currently the most reliable radiological sign of CSI. ${ }^{7,821,26,29}$ However, this is usually seen late in the course.

ABBREVIATIONS AUC = area under the curve; CISS = constructive interference in steady state; CSI = cavernous sinus invasion; GTR = gross-total resection; ICA = internal carotid artery; ROC = receiver operating characteristic; VIBE = volume-interpolated breath-hold examination.

SUBMITTED July 29, 2017. ACCEPTED September 11, 2017.

INCLUDE WHEN CITING Published online March 23, 2018; DOI: 10.3171/2017.9.JNS171699. 
To improve preoperative evaluation of CSI, Knosp and colleagues developed a classification system based on contrast-enhanced T1-weighted MRI scans. ${ }^{21}$ The ICAs were used as landmarks in this classification system to help grade the extent of parasellar extension and determine the probability of intraoperative CSI. The likelihood of CSI increases with higher Knosp grade. The grading system was reevaluated using modern endoscopic techniques and modified, and grade 3 was subdivided into $3 \mathrm{~A}$ and $3 \mathrm{~B} .{ }^{24}$

With advancements in MRI technology, there are now imaging sequences that provide better visualization of the pituitary and sellar anatomy. One such sequence is constructive interference in steady state (CISS), which is a T2weighted gradient echo MRI sequence. ${ }^{14,33,38}$ The CISS sequence provides better visualization of the cranial nerves and cisternal spaces as well as sellar pathologies due to its ability to accentuate T2-weighted values between CSF and surrounding structures. ${ }^{5,16,22,35,37}$ Moreover, the CISS sequence has been demonstrated to be superior to standard T1- and T2-weighted sequences for delineating key structures around the sellar region, including the optic nerve, optic chiasms, oculomotor nerve, pituitary stalk, and small vessels, thus making it more useful for preoperative planning. ${ }^{36}$

While using CISS MRI to evaluate CSI by pituitary macroadenomas has been studied previously, prior work focused on radiological comparisons to only contrastenhanced T1-weighted images without comparison with intraoperative findings. ${ }^{33}$ Thus, we sought to investigate whether CISS MRI is superior to contrast-enhanced T1weighted MRI for the evaluation of CSI by pituitary macroadenomas, using intraoperative evidence of invasion as the gold standard for comparison.

\section{Methods \\ Patients}

This retrospective study was approved by the Cleveland Clinic Institutional Review Board. Data obtained for consecutively treated patients who underwent endoscopic transsphenoidal resection by the staff neurosurgeon (P.F.R.) between February 2014 and June 2016 were retrospectively analyzed. Of the 150 patients identified, 52 patients were excluded due to prior pituitary surgeries or a diagnosis of sellar pathology other than pituitary adenoma. Ninety-eight patients who had a confirmed diagnosis of a pituitary macroadenoma (size $>10 \mathrm{~mm}$ ) and who underwent preoperative CISS and contrast-enhanced T1-weighted volume-interpolated breath-hold examination (VIBE) MRI were included in the study.

\section{MRI Studies}

All MRI scans were acquired using a 1.5-T scanner (Aera, Siemens). Two imaging sequences were included in the study: VIBE and CISS. VIBE is the T1-weighted MRI sequence against which the CISS sequence was compared. The VIBE sequence reported in this study was performed after contrast administration $(0.05 \mathrm{mmol} / \mathrm{kg}$ of intravenous $\mathrm{Gd}$ ). Axial postcontrast VIBE images were acquired with a TR/TE of $9 \mathrm{msec} / 1.79 \mathrm{msec}, 256 \times 256$ matrix, 200-mm field of view, 0.8-mm-thick interleaved slices without a gap, $9^{\circ}$ flip angle, $20 \%$ distance factor, and approximately 4.9 minutes of scan time. Axial CISS images were obtained with a TR/TE of $5.84 \mathrm{msec} / 2.67 \mathrm{msec}$, $256 \times 256$ matrix, 200-mm field of view, 0.7-mm-thick interleaved slices without a gap, $42^{\circ}$ flip angle, $20 \%$ distance factor, and approximately 6.1 minutes of scan time. Coronal reconstructions were made from the volumetric VIBE and CISS axial images.

\section{Pituitary Macroadenoma Analysis}

Pituitary macroadenomas were retrospectively analyzed in a total of 98 patients. A Knosp grade was assigned to each side of the pituitary macroadenoma based on the degree of parasellar extension observed on the VIBE and CISS images. True CSI was determined intraoperatively. In this study, $0^{\circ}$ and $30^{\circ}$ endoscopes were used intraoperatively to inspect both cavernous sinuses for invasion by the macroadenoma. In all patients, the diagnosis of pituitary adenoma was confirmed histologically from a biopsy specimen.

\section{Knosp Grading}

For each cavernous sinus, 3 lines (medial, cross-sectional, and lateral) were drawn on the coronal MR image between the ipsilateral intracavernous and supracavernous portions of the ICA, thereby dividing the parasellar extension into 4 compartments. A grade was given to each side based on the extent of parasellar expansion according to the prior work by Knosp and colleagues (Fig. 1).21,24 The observers (M.L. and D.S.) were trained by the staff neurosurgeon (P.F.R.) in Knosp grading using CISS and VIBE sequence images. M.L. and D.S. each independently graded the extent of the parasellar extension of the pituitary macroadenomas on nonrandomized sequential CISS and VIBE MR images because this method resembled that of the true clinical workflow. However, to minimize potential grading bias, the observers were blinded to the intraoperative findings and postoperative outcomes of all patients. Any discrepancies between the observers were re-reviewed with a third observer (P.F.R.) and resolved by consensus. The subsequent Knosp grades were then compared with the intraoperative findings of whether CSI was present or not.

\section{Intraoperative Determination of CSI}

Patients underwent a standard endoscopic endonasal binostril approach for resection of the pituitary tumor. After tumor resection of the sellar portion, the bilateral cavernous sinus walls were then inspected. The cavernous sinus walls were inspected using a $30^{\circ}$ endoscope, and $45^{\circ}$ and $70^{\circ}$ endoscopes were used if the cavernous walls could not be adequately seen. Lateral exposure was maximized so compression could be distinguished from CSI. Determination of CSI was performed in a manner similar to that described in previous reports. ${ }^{21,24}$ Invasion of the tumor into the cavernous sinus was judged by the operating neurosurgeon (P.F.R.) under direct endoscopic visualization, and the findings were included in the operative reports. The tumor was considered to be invasive if it was observed directly within the cavernous sinus, if it 

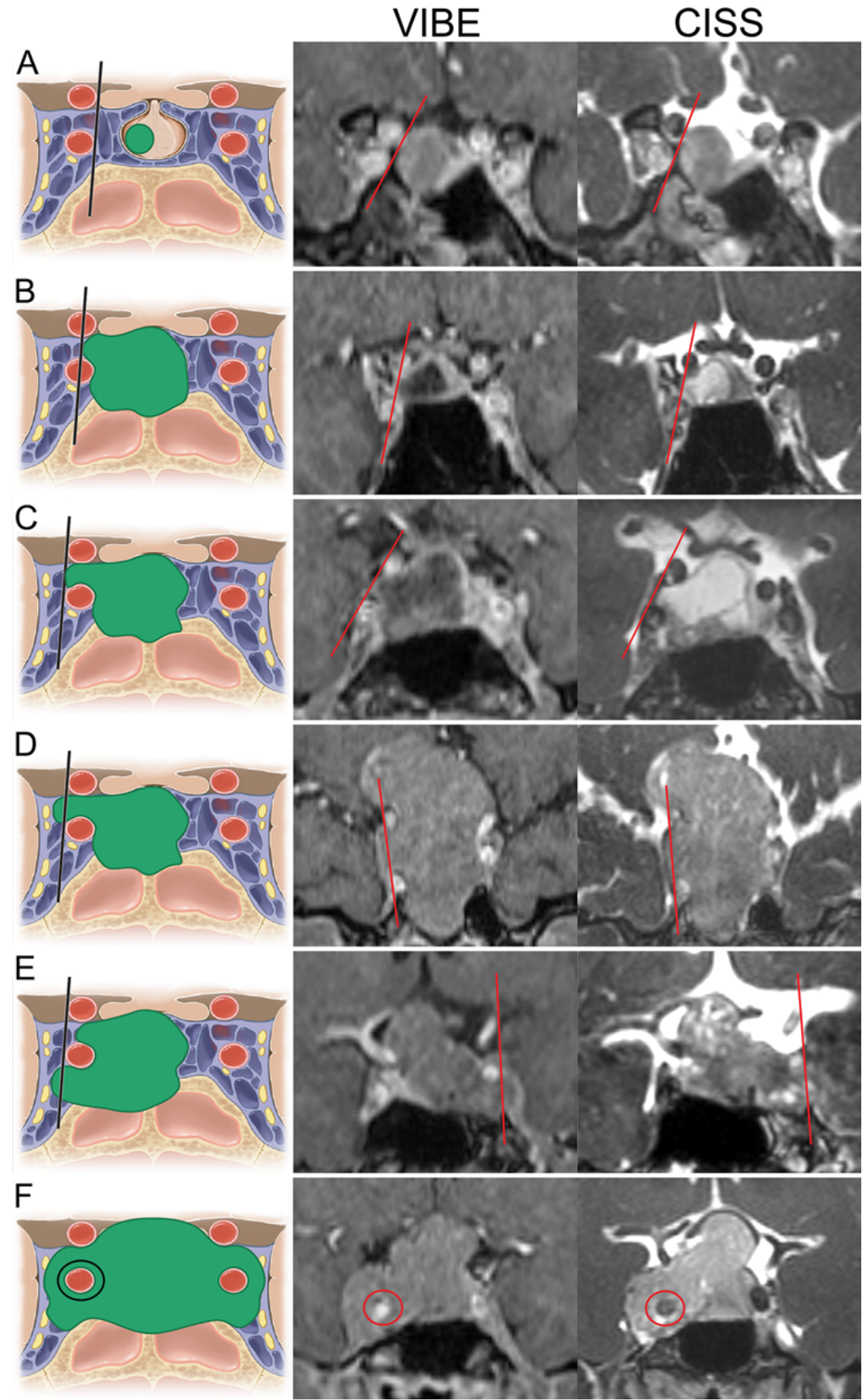

FIG. 1. Left: Graphic illustration of the Knosp classification system for pituitary macroadenomas. Artist: Joe Pangrace. Copyright Center for Medical Art and Photography, Cleveland Clinic Foundation. Published with permission. Center: Representative coronal VIBE images. Right: Representative coronal CISS images. A: Grade 0. The adenoma is medial to the cavernous sinus and does not pass the medial tangent (line), which passes between the medial aspects of the supra- and intracavernous portions of the ICAs. B: Grade 1. The adenoma passes the medial tangent but does not pass the cross-sectional line (line) drawn between the supra- and intracavernous ICAs. C: Grade 2. The adenoma extends beyond the cross-sectional line but does not pass the lateral tangent (line) drawn between the lateral aspects of the supra- and intracavernous ICAs. FIG. 1. (continued) $\rightarrow$ 
FIG. 1. D: Grade 3A. The adenoma extends beyond the lateral tangent (line) and into the superior cavernous sinus compartment. E: Grade 3B. The adenoma extends beyond the lateral tangent (line) and into the inferior cavernous sinus compartment. F: Grade 4. The adenoma completely encases the intracavernous ICA (circle). Figure is available in color online only.

wrapped completely around the intracavernous portion of the ICA, or if the adventitia of the intracavernous portion of the ICA was visible. If the medial wall of the cavernous sinus was smooth and intact after tumor debulking, CSI was deemed to be absent.

\section{Statistical Analysis}

RStudio was used for the statistical analysis. Categorical variables are summarized as frequencies and percentages and were compared using the chi-square test. Continuous variables are presented as the median and 25th-75th percentiles because these variables are not normally distributed; these variables were compared using the Wilcoxon rank-sum test. The pairwise Spearman correlation coefficient was calculated among observers to describe grading reliability. Statistical significance was set as $\mathrm{p}<0.05$.

The predictive accuracy of CISS- versus VIBE-derived Knosp grades is represented graphically by the receiver operating characteristic (ROC) curves. The ROC curve is a plot of the true positive rate (sensitivity) versus the false positive rate $(1-$ specificity) of each Knosp grade. The area under the curve (AUC) was used to compare CISSand VIBE-derived Knosp grades with bootstrap analysis after 2000 samplings with replacement. The bootstrap distributions of the CISS and VIBE AUCs are reported as 95\% CI values. The pROC package for RStudio was used for this part of the analysis. ${ }^{28}$

\section{Results}

The baseline patient characteristics, including sex, age, tumor diameter, and tumor type, are presented in Table 1 . The median diameter of the pituitary macroadenomas was $26 \mathrm{~mm}$. The majority (77\%) of the pituitary macroad-

TABLE 1. Baseline patient and pituitary adenoma characteristics

\begin{tabular}{lc}
\hline \multicolumn{1}{c}{ Characteristic } & Value \\
\hline Patient & \\
\hline Sex, no. & 46 \\
\hline Male & 52 \\
\hline Female & $57(43-67)$ \\
\hline Median age (IQR), yrs \\
\hline Adenoma \\
\hline Median diameter (IQR), mm \\
\hline Hormone secretion, no. (\%) \\
\hline Nonfunctioning \\
\hline Prolactin \\
\hline Adrenocorticotropic hormone \\
\hline Growth hormone & $75(77)$ \\
\hline Thyroid-stimulating hormone & $7(6)$ \\
\hline
\end{tabular}

$\mathrm{IQR}=$ interquartile range. enomas were nonfunctioning, while $6 \%$ were prolactin secreting, $7 \%$ were adrenocorticotropic hormone secreting, $9 \%$ were growth hormone secreting, and $1 \%$ were thyroidstimulating hormone secreting (Table 1).

Twenty-seven (27.6\%) of 98 pituitary macroadenomas were found to invade the cavernous sinus intraoperatively. The size of the pituitary macroadenomas with CSI was greater than that of those without CSI (median [25th-75th percentile] $29 \mathrm{~mm}$ [23-34 mm] vs $25 \mathrm{~mm}$ [18-30 mm], respectively), but the difference was not statistically significant $(\mathrm{p}=0.081)$.

Both sides of each pituitary macroadenoma were graded. The image resolution of the CISS sequences was superior to that of the VIBE sequences because the ICAs and the dural borders that surround the pituitary were more easily visualized on the CISS images (Figs. 1-3). In 2 cases, while the pituitary macroadenoma seemed to fully encase the ICA (Knosp grade 4) on the VIBE images, the CISS images did not suggest complete encasement and resulted in lower Knosp grades of 3A and 2 (Fig. 2). For these 2 patients, there were no intraoperative findings of CSI.

When the parasellar extensions of the pituitary macroadenomas on VIBE images were correlated to the intraoperative findings, $0.0 \%$ of Knosp grade $0,4.5 \%$ of Knosp grade 1, 23.8\% of Knosp grade 2, 42.1\% of Knosp grade $3 \mathrm{~A}, 100 \%$ of Knosp grade 3B, and $83.3 \%$ of Knosp grade 4 adenomas were found to have CSI. In contrast, when the Knosp grades determined using the CISS images were
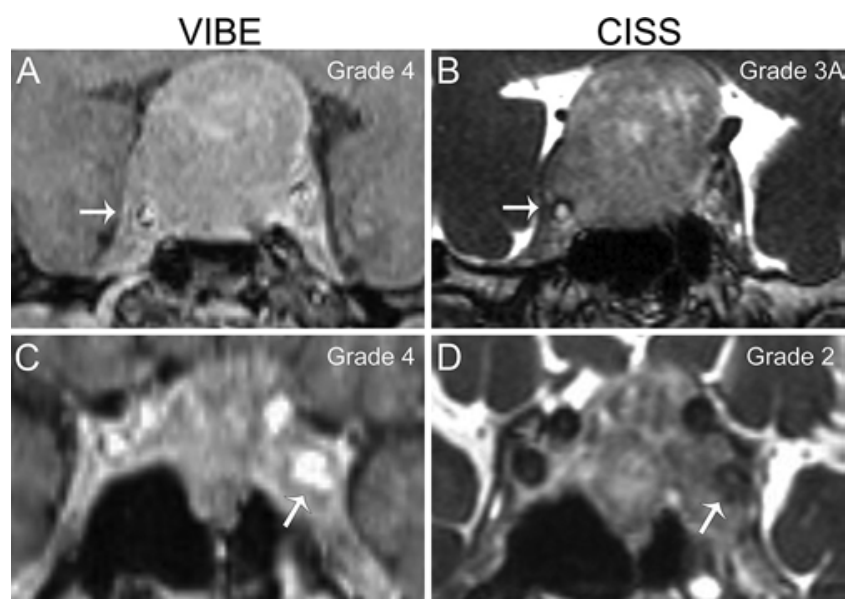

FIG. 2. Two cases of pituitary macroadenoma classified as Knosp grade 4 on VIBE images but without intraoperative findings of CSI. A and B: In the first case, the pituitary macroadenoma appears to fully encase the intracavernous portion of the right ICA (arrows) on VIBE imaging. However, with better visualization of the dural boundaries and the outline of the macroadenoma on CISS imaging, it was clear that the pituitary macroadenoma did not encase the ICA but rather extended into the superior cavernous sinus compartment. CSI was not observed on the right side intraoperatively. C and D: In the second case, the pituitary macroadenoma appears to fully encase the left ICA (arrows) on VIBE imaging but not on CISS imaging. CSI was not observed intraoperatively on the left side. 


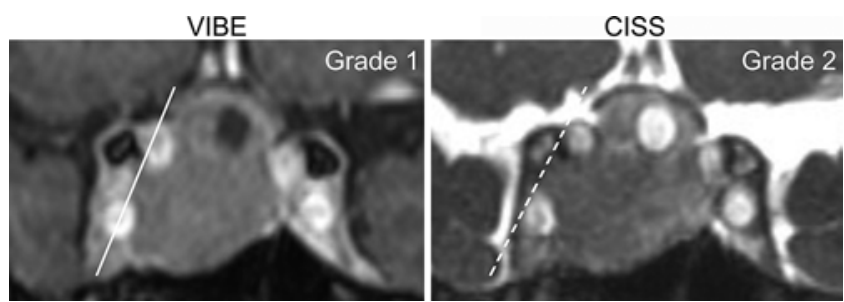

FIG. 3. Example of a pituitary macroadenoma with a lower Knosp grade on VIBE (left) than CISS (right) images. The ICAs, pituitary macroadenoma, and sellar anatomy were better visualized on CISS images. The pituitary macroadenoma did not extend past the solid cross-sectional line on the VIBE image (Knosp grade 1) but reached the dashed lateral tangent line on the CISS image (Knosp grade 2). CSI was not observed intraoperatively.

used, $0.0 \%$ of grade $0,2.1 \%$ of grade $1,31.3 \%$ of grade $2,56.3 \%$ of grade $3 \mathrm{~A}, 100 \%$ of grade $3 \mathrm{~B}$, and $100 \%$ of grade 4 adenomas were found be invasive intraoperatively (Table 2; Fig. 4). A subanalysis of only nonfunctioning pituitary macroadenomas showed a similar correlation between Knosp grade and the rate of CSI (Supplemental Table 1 and Supplemental Fig. 1).

If grades $3 \mathrm{~A}, 3 \mathrm{~B}$, and 4 were considered to be "invasive grades," the sensitivity and specificity were $74.1 \%$ and $92.3 \%$ for the VIBE sequences, respectively, and $77.8 \%$ and $95.9 \%$ for the CISS sequences, respectively. Of 196 assessments (both the left and right sides of 98 macroadenomas), 45 cases (23.0\%) had different Knosp grades when scored using VIBE versus CISS images. Consistent with prior reports, CSI prevalence increased with higher Knosp grade, regardless of which imaging sequence was used (Fig. 4). Overall, the interobserver reliability was strong and similar to that reported for prior studies $(0.71){ }^{25,33}$

ROC curves were constructed to compare the diagnostic value of the Knosp grades determined using the CISS and VIBE sequences for predicting CSI (Fig. 5). The AUC of the CISS curve was significantly greater than that of the VIBE curve $(0.97$ [95\% CI $0.95-0.99$ ] vs 0.94 [95\% CI 0.90-0.97], respectively; $p=0.007$ ). This suggests that the Knosp grades determined using the CISS sequence images are better correlated with the intraoperative finding of CSI and have better predicative accuracy for CSI. The subanalysis that included only nonfunctioning pitu-

TABLE 2. CSI according to the Knosp grades derived from the CISS and VIBE sequence images

\begin{tabular}{cccrcc}
\hline & \multicolumn{4}{c}{ No. of Sides (\%) } \\
\cline { 2 - 3 } \cline { 5 - 5 } $\begin{array}{c}\text { Knosp } \\
\text { Grade }\end{array}$ & \multicolumn{2}{c}{ VIBE } & & \multicolumn{2}{c}{ CISS } \\
\cline { 2 - 3 } \cline { 5 - 6 } & Total & CSI & & Total & CSI \\
\hline 0 & 98 & $0(0.0)$ & & 104 & $0(0.0)$ \\
\hline 1 & 44 & $2(4.5)$ & & 48 & $1(2.1)$ \\
\hline 2 & 21 & $5(23.8)$ & & 16 & $5(31.3)$ \\
\hline $3 A$ & 19 & $8(42.1)$ & & 16 & $9(56.3)$ \\
\hline $3 B$ & 2 & $2(100.0)$ & & 2 & $2(100.0)$ \\
\hline 4 & 12 & $10(83.3)$ & 10 & $10(100.0)$ \\
\hline
\end{tabular}

Both the left and right sides of the pituitary adenomas (98 tumors with 196 sides) were intraoperatively graded and assessed for CSI.

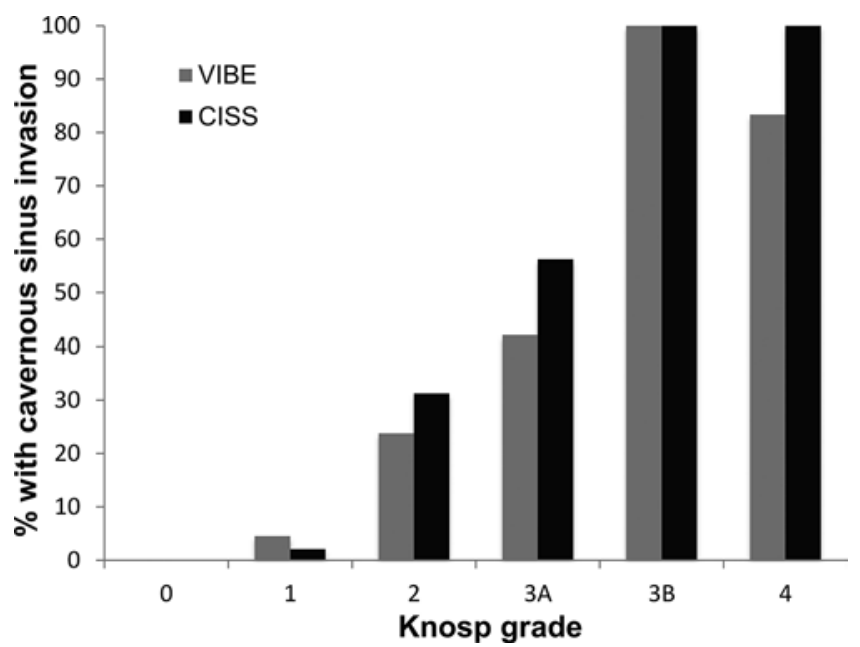

FIG. 4. Intraoperative findings of CSI plotted against Knosp grades determined using CISS (black bars) and VIBE (gray bars) images. The prevalence of CSI increased with higher Knosp grade, regardless of whether CISS or VIBE images were used for grading. While $100 \%$ of grade 4 macroadenomas classified using CISS sequence images were found to be invasive, only $83.3 \%$ of grade 4 macroadenomas classified using VIBE sequence images were found to be invasive.

itary macroadenomas also demonstrated that the AUC of the CISS curve was significantly greater than that of the VIBE curve (0.96 [95\% CI 0.92-0.99] vs 0.91 [95\% CI 0.86-0.96], respectively; $p=0.006$ ) (Supplemental Fig. 2).

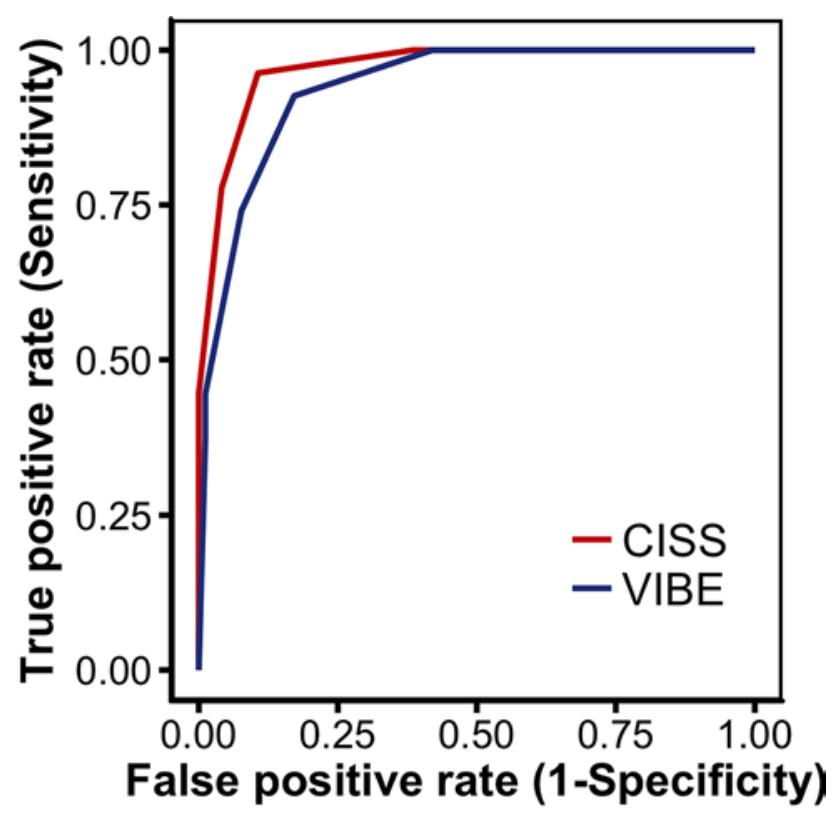

\begin{tabular}{c|ccc} 
& VIBE & CISS & $p$ Value \\
\hline $\begin{array}{c}\text { Area Under } \\
\text { Curve }\end{array}$ & $\mathbf{0 . 9 4}$ & 0.97 & $\mathbf{0 . 0 0 7}$ \\
$(95 \% \mathrm{Cl}: 0.90-0.97)$ & $(95 \% \mathrm{Cl}: 0.95-0.99)$ &
\end{tabular}

FIG. 5. ROC curves of Knosp grades derived from CISS (red line) and VIBE (blue line) imaging. The points on the plot were obtained by setting each Knosp grade as the threshold for the diagnosis of CSI. The AUC was significantly greater for the CISS ROC curve than the VIBE ROC curve $(0.97$ vs $0.94 ; p=0.007)$. Figure is available in color online only. 
TABLE 3. Rates of GTR according to the Knosp grades derived from the CISS and VIBE images

\begin{tabular}{ccccccc}
\hline & \multicolumn{4}{c}{ No. of Patients (\%) } & \\
\cline { 2 - 3 } $\begin{array}{c}\text { Knosp } \\
\text { Grade }\end{array}$ & \multicolumn{2}{c}{ VIBE } & & \multicolumn{2}{c}{ CISS } & Total \\
\cline { 2 - 3 } \cline { 5 - 6 } & No. of GTRs & & Total & No. of GTRs & Value \\
\hline 0 & 24 & $20(83.3)$ & & 31 & $26(83.9)$ & 0.812 \\
\hline 1 & 31 & $28(90.3)$ & & 27 & $25(92.6)$ & 0.886 \\
\hline 2 & 15 & $14(93.3)$ & & 15 & $13(86.7)$ & 0.903 \\
\hline $3 A$ & 15 & $12(80.0)$ & & 14 & $11(78.6)$ & 0.751 \\
\hline $3 B$ & 2 & $1(50.0)$ & & 2 & $1(50.0)$ & 1.000 \\
\hline 4 & 11 & $5(45.5)$ & & 9 & $3(33.3)$ & 0.759 \\
\hline
\end{tabular}

The sample size is the total number of patients $(n=98)$. No significant differences were observed between the rates of GTR at any Knosp grade derived from CISS and VIBE images.

The percentage of cases with GTR decreased with increasing Knosp grade, regardless of which imaging sequence was used (Table 3). For high-grade (3A, 3B, and 4) pituitary macroadenomas classified using VIBE and CISS sequences, the rates of GTR were $64.3 \%$ and $60.0 \%$, respectively. Overall, the percentage of patients with GTR was not significantly different at any Knosp grade derived using CISS and VIBE images (Table 3).

\section{Discussion}

In the prior studies by Knosp and colleagues, radiological assessment of CSI was based on Gd-enhanced T1-weighted MR images. ${ }^{21,24}$ However, T1-weighted MRI might not be the best MRI sequence for evaluating pituitary pathologies. T2-weighted sequences have been shown to be superior for the evaluation of the sellar region. ${ }^{4}$ In fact, Knosp and colleagues stated in their article that high-resolution T2-weighted MRI better demonstrated CSI than T1-weighted imaging in some cases, but this was not formally evaluated. ${ }^{24}$ With newer high-resolution MRI sequences being developed, the imaging capabilities for pituitary and sellar pathologies have improved. ${ }^{6}$ Therefore, the imaging modality for Knosp grading should be updated to reflect advancements in MRI technology.

In our patient cohort, the Knosp grades determined from the CISS sequences were overall better correlated with the risk of CSI than the VIBE sequences. This might be due to increased overlap between gadolinium enhancement of the tumor and blood in the cavernous sinus on VIBE images, whereas contrast was not used for CISS imaging. The comparison between the ROC curves and AUCs provided further confirmation of the CISS sequence's superiority (Fig. 5).

While our study was not the first to report the use of CISS sequences to evaluate pituitary adenomas, the sample size was much more robust in our study than in 2 prior studies. ${ }^{33,36}$ Similar to our observations, Xie et al. demonstrated CISS sequence's superiority over standard T1- and T2-weighted images for delineating fine sellar structures, including the optic nerve, optic chiasm, oculomotor nerve, infundibulum, pituitary stalk, and small vessels. ${ }^{36}$ CISS appeared to be valuable for the preoperative treatment planning for midline skull base lesions and preventing un- necessary damage to the sellar structures. Tong et al. drew similar conclusions because they also found CISS MRI was, on average, good to excellent as a tool for evaluating CSI by pituitary macroadenomas. ${ }^{33}$ There were limitations of their study that require the results to be interpreted with caution and limit comparison between their study and ours. First, the grades obtained using contrast-enhanced T1-weighted imaging were set as the standard for comparison rather than intraoperative findings. Given that direct intraoperative observation of CSI is regarded as the gold standard rather than imaging, it is not possible to reliably conclude which imaging sequence was actually superior. Second, the Knosp grades obtained using T1-weighted images were compared against a different, newly devised grading system using CISS. Comparing 2 different grading systems on different imaging sequences limits the interpretability of the results..$^{33}$

The Knosp grades determined using the CISS images were more conservative than those obtained using VIBE images because fewer pituitary macroadenomas were classified as grade 4 (Table 2). More importantly, the percentages of macroadenomas classified as grade 4 by VIBE and CISS sequences that were found to have CSI intraoperatively were $83.3 \%$ and $100 \%$, respectively. In fact, this is the first report of 2 Knosp grade 4 pituitary macroadenomas classified using a contrast-enhanced T1-weighted MRI that were not found to show CSI intraoperatively. CISS sequences, however, provided superior visualization of the pituitary and sellar anatomy in both cases and we did not classify the macroadenomas as grade 4 because full encasement of the intracavernous portion of the ICA was not observed. Furthermore, intraoperative inspection with both $0^{\circ}$ and $30^{\circ}$ endoscopes found no obvious signs of CSI, and complete resection of all visible tumor was achieved in both cases (Fig. 2). The lack of previous reports of Knosp grade 4 macroadenomas without CSI that were classified using T1-weighted sequences may be due to several reasons: improved visualization of the sellar region with modern endoscopic techniques, potential variability in the grading of the macroadenomas between groups, and subjective intraoperative observation by the operating surgeon..$^{25}$ Nevertheless, our results suggest that not all Knosp grade 4 macroadenomas, at least those graded using T1-weighted MRI sequences, are associated with CSI. Whether Knosp grade 4 macroadenomas classified using CISS MRI are $100 \%$ predictive of CSI requires further investigation.

A recent meta-analysis showed that Knosp grades 3-4 are the best objective indicators for CSI. ${ }^{11}$ When we considered grades $3 \mathrm{~A}, 3 \mathrm{~B}$, and 4 to be invasive, the sensitivity and specificity was better for CISS sequences $(77.8 \%$ and $95.9 \%$, respectively) than VIBE sequences $(74.1 \%$ and $92.3 \%$, respectively). Micko et al. found that grade 3B macroadenomas led to significantly more cases of CSI than grade 3A, but a similar conclusion cannot be definitively drawn from our study because of the small number of grade $3 \mathrm{~B}$ macroadenomas. ${ }^{24}$ However, our results do suggest that grade $3 \mathrm{~A}$ should remain as a high-risk grade for potential CSI because the sensitivity drops by approximately $30 \%$ if only grades 3B and 4 were considered to be invasive, regardless of which imaging sequence was used. 
The gold standard for the diagnosis of CSI is direct intraoperative observation of invasion. Although this is the most precise method to date, it does depend on the operating neurosurgeon and, as such, could be a potential source of error in this study. The overall percentage of CSI determined by Knosp grade found in our study was similar to that reported in previous studies. ${ }^{11,21,24}$ Although we found slightly higher rates of CSI in Knosp grade 2 and 3A cases than were found in the original study by Micko et al., our sample size was too small to make a meaningful statistical comparison. ${ }^{24}$ Obtaining a histological sample from the medial dural wall can also confirm CSI. This is not routinely performed, however, given the close proximity of the ICA, technical difficulty, and risk of neurovascular injuries. ${ }^{23,24,32,39}$

Since the adoption of the endoscopic approach as the preference over microscopy, GTR rates have improved due to the endoscope's capacity to show panoramic and angled views of the sellar space. However, the reported rates of GTR of pituitary macroadenomas with CSI vary tremendously among different groups and range from $0 \%$ to $92.3 \%{ }^{2,3,9,10,19,24}$ This variability may be partly due to the differing levels of experience among operating surgeons. ${ }^{11}$ In our study, the GTR rate decreased with higher Knosp grade, regardless of whether the Knosp grades were derived from VIBE or CISS images. The GTR rate of Knosp grade 3-4 pituitary macroadenomas using the endoscopic approach was $47 \%$ in a recent meta-analysis, which is consistent with the GTR rate reported in our patient cohort. ${ }^{26}$

The utility of CISS sequences is not limited to neuronavigation and preoperative planning. With the CISS sequence's superior ability to delineate cranial nerves and small vessels within the cavernous sinus, its use in radiosurgery for skull base tumors can improve the precision of stereotactic irradiation and preserve the surrounding vital structures. ${ }^{17,27}$ Although a decrease in radiosurgeryrelated complications is expected given the more precise targeting, long-term follow-up data are currently lacking. Furthermore, CISS imaging is also useful as an adjunct sequence to T1-weighted MRI for image registration before radiosurgery for trigeminal neuralgia. ${ }^{34,40}$ These encouraging results of CISS MRI thus warrant further investigation of this sequence in different neurosurgical settings.

However, there are several limitations to our study. First, the retrospective nature of this study has inherent limitations-surgeon bias and referral bias may exist, and the subset analysis is limited. Second, the sample size was relatively small for high Knosp grades (3A, 3B, and 4). Future studies with large cohorts of high Knosp grade pituitary macroadenomas are needed to better determine the correlation between Knosp grade and CSI. Third, we recognize that Knosp grading is associated with interrater variability and is a source of inaccuracy ${ }^{25,33}$ Interrater differences were observed in our study, but the overall correlation between observers was strong (0.71) and consistent with previous reports..$^{25,33}$ While we attempted to improve interrater reliability by including a third observer to reconcile discrepant grades, it nevertheless remains a limitation of our study. Finally, CISS sequencing is not available at all institutions and may require sufficient familiarity with the sequences.

\section{Conclusions}

Our results suggest that Knosp grades determined from CISS sequences are more useful than those determined from VIBE sequences when evaluating CSI by pituitary macroadenomas. CISS MRI may have a primary role in the preoperative workup of pituitary macroadenomas for surgical planning and patient counseling.

\section{Acknowledgments}

We thank the medical illustrator, Joe Pangrace, for the graphic illustrations of the Knosp grades.

\section{References}

1. Ahmadi J, North CM, Segall HD, Zee CS, Weiss MH: Cavernous sinus invasion by pituitary adenomas. AJR Am J Roentgenol 146:257-262, 1986

2. Alahmadi H, Dehdashti AR, Gentili F: Endoscopic endonasal surgery in recurrent and residual pituitary adenomas after microscopic resection. World Neurosurg 77:540-547, 2012

3. Bao X, Deng K, Liu X, Feng M, Chen CC, Lian W, et al: Extended transsphenoidal approach for pituitary adenomas invading the cavernous sinus using multiple complementary techniques. Pituitary 19:1-10, 2016

4. Bladowska J, Biel A, Zimny A, Lubkowska K, BednarekTupikowska G, Sozanski T, et al: Are T2-weighted images more useful than T1-weighted contrast-enhanced images in assessment of postoperative sella and parasellar region? Med Sci Monit 17:MT83-MT90, 2011

5. Blitz AM, Macedo LL, Chonka ZD, Ilica AT, Choudhri AF, Gallia GL, et al: High-resolution CISS MR imaging with and without contrast for evaluation of the upper cranial nerves: segmental anatomy and selected pathologic conditions of the cisternal through extraforaminal segments. Neuroimaging Clin N Am 24:17-34, 2014

6. Chaudhary V, Bano S: Imaging of the pituitary: recent advances. Indian J Endocrinol Metab 15 (Suppl 3): S216S223, 2011

7. Cottier JP, Destrieux C, Brunereau L, Bertrand P, Moreau L, Jan M, et al: Cavernous sinus invasion by pituitary adenoma: MR imaging. Radiology 215:463-469, 2000

8. Cukiert A, Andrioli M, Goldman J, Nery M, Salgado L, Knoepfelmacher M, et al: Cavernous sinus invasion by pituitary macroadenomas. Neuroradiological, clinical and surgical correlation. Arq Neuropsiquiatr 56:107-110, 1998

9. Dehdashti AR, Ganna A, Karabatsou K, Gentili F: Pure endoscopic endonasal approach for pituitary adenomas: early surgical results in 200 patients and comparison with previous microsurgical series. Neurosurgery 62:1006-1017, 2008

10. Dehdashti AR, Gentili F: Current state of the art in the diagnosis and surgical treatment of Cushing disease: early experience with a purely endoscopic endonasal technique. Neurosurg Focus 23(3):E9, 2007

11. Dhandapani S, Singh H, Negm HM, Cohen S, Anand VK, Schwartz TH: Cavernous sinus invasion in pituitary adenomas: systematic review and pooled data meta-analysis of radiologic criteria and comparison of endoscopic and microscopic surgery. World Neurosurg 96:36-46, 2016

12. Fahlbusch R, Buchfelder M: Transsphenoidal surgery of parasellar pituitary adenomas. Acta Neurochir (Wien) 92:93-99, 1988

13. Frank G, Pasquini E: Endoscopic endonasal cavernous sinus surgery, with special reference to pituitary adenomas. Front Horm Res 34:64-82, 2006

14. Fushimi Y, Miki Y, Ueba T, Kanagaki M, Takahashi T, Yamamoto A, et al: Liliequist membrane: three-dimensional constructive interference in steady state MR imaging. Radiology 229:360-365, 2003 
15. Giovanelli M, Losa M, Mortini P: Surgical therapy of pituitary adenomas. Metabolism 45 (8 Suppl 1):115-116, 1996

16. Hatipoğlu HG, Durakoğlugil T, Ciliz D, Yüksel E: Comparison of FSE T2W and 3D FIESTA sequences in the evaluation of posterior fossa cranial nerves with MR cisternography. Diagn Interv Radiol 13:56-60, 2007

17. Hayashi M, Chernov MF, Tamura N, Yomo S, Tamura M, Horiba A, et al: Usefulness of the advanced neuroimaging protocol based on plain and gadolinium-enhanced constructive interference in steady state images for Gamma Knife radiosurgery and planning microsurgical procedures for skull base tumors. Acta Neurochir Suppl 116:167-178, 2013

18. Jho HD: Endoscopic transsphenoidal surgery. J Neurooncol 54:187-195, 2001

19. Jho HD, Carrau RL: Endoscopic endonasal transsphenoidal surgery: experience with 50 patients. J Neurosurg 87:44-51, 1997

20. Kabil MS, Eby JB, Shahinian HK: Fully endoscopic endonasal vs. transseptal transsphenoidal pituitary surgery. Minim Invasive Neurosurg 48:348-354, 2005

21. Knosp E, Steiner E, Kitz K, Matula C: Pituitary adenomas with invasion of the cavernous sinus space: a magnetic resonance imaging classification compared with surgical findings. Neurosurgery 33:610-618, 1993

22. Kulkarni M: Constructive interference in steady-state/FIESTA-C clinical applications in neuroimaging. J Med Imaging Radiat Oncol 55:183-190, 2011 (Erratum in J Med Imaging Radiat Oncol 55:452, 2011)

23. Messerer M, Dubourg J, Saint-Pierre G, Jouanneau E, Sindou M: Percutaneous biopsy of lesions in the cavernous sinus region through the foramen ovale: diagnostic accuracy and limits in 50 patients. J Neurosurg 116:390-398, 2012

24. Micko AS, Wöhrer A, Wolfsberger S, Knosp E: Invasion of the cavernous sinus space in pituitary adenomas: endoscopic verification and its correlation with an MRI-based classification. J Neurosurg 122:803-811, 2015

25. Mooney MA, Hardesty DA, Sheehy JP, Bird R, Chapple K, White WL, et al: Interrater and intrarater reliability of the Knosp scale for pituitary adenoma grading. J Neurosurg 126:1714-1719, 2017

26. Moreau L, Cottier JP, Bertrand P, Destrieux C, Jan M, Sonier $\mathrm{CB}$, et al: [MRI diagnosis of sinus cavernous invasion by pituitary adenomas.] J Radiol 79:241-246, 1998 (Fr)

27. Régis J, Tamura M, Wikler D, Porcheron D, Levrier O: Radiosurgery: operative technique, pitfalls and tips. Prog Neurol Surg 21:54-64, 2008

28. Robin X, Turck N, Hainard A, Tiberti N, Lisacek F, Sanchez JC, et al: pROC: an open-source package for R and $\mathrm{S}+$ to analyze and compare ROC curves. BMC Bioinformatics 12:77, 2011

29. Scotti G, Yu CY, Dillon WP, Norman D, Colombo N, Newton $\mathrm{TH}$, et al: MR imaging of cavernous sinus involvement by pituitary adenomas. AJR Am J Roentgenol 151:799-806, 1988

30. Selman WR, Laws ER Jr, Scheithauer BW, Carpenter SM: The occurrence of dural invasion in pituitary adenomas. $\mathbf{J}$ Neurosurg 64:402-407, 1986

31. Shou XF, Li SQ, Wang YF, Zhao Y, Jia PF, Zhou LF: Treatment of pituitary adenomas with a transsphenoidal approach. Neurosurgery 56:249-256, 2005

32. Sindou M, Chavez JM, Saint Pierre G, Jouvet A: Percutaneous biopsy of cavernous sinus tumors through the foramen ovale. Neurosurgery 40:106-111, 1997
33. Tong T, Yue W, Zhong Y, Zhenwei Y, Yong H, Xiaoyuan F: Comparison of contrast-enhanced SPACE and CISS in evaluating cavernous sinus invasion by pituitary macroadenomas on 3-T magnetic resonance. J Comput Assist Tomogr 39:222-227, 2015

34. Wang TJ, Brisman R, Lu ZF, Li X, Isaacson SR, Shah JN, et al: Image registration strategy of $\mathrm{T}_{1}$-weighted and FIESTA MRI sequences in trigeminal neuralgia Gamma Knife radiosurgery. Stereotact Funct Neurosurg 88:239-245, 2010

35. Watanabe Y, Makidono A, Nakamura M, Saida Y: 3D MR cisternography to identify distal dural rings: comparison of 3D-CISS and 3D-SPACE sequences. Magn Reson Med Sci 10:29-32, 2011

36. Xie T, Zhang XB, Yun H, Hu F, Yu Y, Gu Y: 3D-FIESTA MR images are useful in the evaluation of the endoscopic expanded endonasal approach for midline skull-base lesions. Acta Neurochir (Wien) 153:12-18, 2011

37. Yagi A, Sato N, Taketomi A, Nakajima T, Morita H, Koyama Y, et al: Normal cranial nerves in the cavernous sinuses: contrast-enhanced three-dimensional constructive interference in the steady state MR imaging. AJNR Am J Neuroradiol 26:946-950, 2005

38. Yamamoto J, Kakeda S, Shimajiri S, Takahashi M, Watanabe $\mathrm{K}$, Kai Y, et al: Tumor consistency of pituitary macroadenomas: predictive analysis on the basis of imaging features with contrast-enhanced 3D FIESTA at 3T. AJNR Am J Neuroradiol 35:297-303, 2014

39. Yi W, Ohman K, Brännström T, Bergenheim AT: Percutaneous biopsy of cavernous sinus tumour via the foramen ovale. Acta Neurochir (Wien) 151:401-407, 2009

40. Zerris VA, Noren GC, Shucart WA, Rogg J, Friehs GM: Targeting the cranial nerve: microradiosurgery for trigeminal neuralgia with CISS and 3D-flash MR imaging sequences. J Neurosurg 102 Suppl:107-110, 2005

\section{Disclosures}

Dr. Recinos is a member of the Scientific Advisory Board of Acera Surgical Inc. and owns stock in Acera Surgical Inc.

\section{Author Contributions}

Conception and design: Recinos, Lang. Acquisition of data: Lang, Silva. Analysis and interpretation of data: Lang, Silva, Dai. Drafting the article: Recinos, Lang, Silva, Dai, Kshettry, Sindwani. Critically revising the article: all authors. Reviewed submitted version of manuscript: all authors. Approved the final version of the manuscript on behalf of all authors: Recinos. Statistical analysis: Lang, Dai. Administrative/technical/material support: Kshettry. Study supervision: Recinos, Kshettry, Woodard, Sindwani.

\section{Online-Only Content}

Supplemental material is available with the online version of the article.

Supplemental Table and Figures. https://thejns.org/doi/suppl/ 10.3171/2017.9.JNS171699.

\section{Correspondence}

Pablo F. Recinos: Rose Ella Burkhardt Brain Tumor \& NeuroOncology Center, Cleveland, OH. recinop@ccf.org. 